\section{Passengers masquerading as cancer drivers}

Analyses of cancer genome sequencing data typically make the assumption that the recurrence of the same mutation across patients is indicative that the mutation is functionally selected for during cancer evolution, and therefore the gene in which it occurs is a likely cancer driver. Although this is certainly one cause of mutation recurrence in cancer genomes, it is unclear if there are other causes that might be involved.

Recent pan-cancer genome analyses have examined mutations at the small scale (the trinucleotide level), identifying mutational signatures of specific carcinogens or endogenous mutational processes, or at the large scale (the megabase level), identifying that topologically associating domains (TADs) in different nuclear compartments are differentially susceptible to mutation. However, little attention has been paid to cancer genomes at the mesoscale ( $20 \mathrm{bp}$ level), which includes features such as DNA hairpins (also known as stem-loops) and other local DNA secondary structures. Buisson et al. investigated whether mutation recurrence is affected by such mesoscale features and found that some mutation hotspots might arise owing to the preference of a mutagen for a particular secondary structure, therefore suggesting that these mutations are most likely passenger mutations masquerading as drivers.

Combining whole genome sequencing (WGS) data from several sources, the authors first analysed mutations at the small and large scale. At the small scale, they observed mutational signatures that were in line with previous results. At the large scale, they confirmed that mutations are more frequent in TADs that are gene-poor, transcriptionally repressed and late replicating, but most known cancer driver genes are found in TADs that are more transcriptionally active, early replicating and less prone to mutations. Combining the two analyses indicated that the mutation frequency of most endogenous and exogenous mutagens is higher in latereplicating DNA, with one exception: mutagenesis driven by APOBEC cytidine deaminases did not depend on replication timing.

The authors then hypothesized that the mutation frequency induced by APOBECs might be influenced by the presence of DNA hairpin structures, in which bases located in the loop tend to 'flip out', potentially increasing their susceptibility to mutagenesis. Indeed, nucleotides in potential hairpin loops, determined by quantifying likely 'stem strength', were more frequently mutated than nucleotides elsewhere in the genome.

Three APOBEC family members (APOBEC3A, APOBEC3B and APOBEC3H haplotype I) have previously been linked to the APOBEC mutational signature (mutation at TpC dinucleotides) in cancer genomes. In vitro assays with extracts from cells expressing one of these three APOBECs and a DNA substrate comprised of either a naturally occurring hairpin of the NUP93 gene or a mutated NUP93 sequence incapable of forming a hairpin indicated that only APOBEC3A had stronger activity on hairpin-forming NUP93. Consistent with this, in the tumour WGS data the mutation frequency of APOBEC3A-dominated samples, but not APOBEC3B-dominated samples, depended on hairpin potential. These data suggest that APOBEC3A is the enzyme most likely responsible for mutations in hairpin loops in cancer genomes.

Further in vitro analyses were then done to refine the preferred substrates of APOBEC3A. APOBEC3A activity was equal on stems ranging in length from $3-7 \mathrm{bp}$ and was highest in stems with the strongest base pairing. The enzyme also exhibited a preference for $\mathrm{TpC}$ sites at the $3^{\prime}$ end of the loop and for smaller loop size ( $<7$ nucleotides). Similar patterns were observed in the tumour WGS data. Furthermore, analysis of the sequence context of mutated $\mathrm{TpC}$ dinucleotides indicated that a YTCR motif (where $\mathrm{Y}=\mathrm{C} / \mathrm{T}$ and $\mathrm{R}=\mathrm{A} / \mathrm{G}$ ) is preferred by $\mathrm{APOBEC} 3 \mathrm{~A}$ in vitro. The YTCR sequence is also enriched at $\mathrm{TpC}$ sites that are recurrently mutated across $\mathrm{APOBEC}^{+}$tumours. Looking at these features of APOBEC3A mutagenesis across whole exome sequencing data from 2,572 $\mathrm{APOBEC}^{+}$human tumours, the authors found that

mutation hotspots tended to fall within two distinct groups. First, 55 of the top 100 most frequently mutated sites were in genes previously described as cancer drivers (for example, PIK3CA and TP53), but all of these except one (in MB21D2) occurred at non-optimal APOBEC3A sites. The second group (45 of 100) comprised recurrent mutations at optimal APOBEC3A sites, but in genes not known to be associated with cancer. The authors propose that these mutations are 'passenger hotspots' that most likely recur in tumours because they are easily generated by APOBEC3A activity. Because the recurrent mutation in MB21D2 also arises at an optimal APOBEC3A site, and there is no strong evidence of its role as a true cancer driver, the authors suggest that it may also these mutations are 'passenger hotspots' that most likely recur in tumours because they are easily generated by APOBEC3A activity 5 be a passenger hotspot. Furthermore, the genes that are recurrently mutated at non-optimal APOBEC3A sites might be as-yet unidentified cancer drivers.

This study emphasizes the importance of analysing the role of mesoscale genome features in generating mutation hotspots, and considering these features when classifying genes as cancer drivers.

Sarah Seton-Rogers

ORIGINAL ARTICLE Buisson, R. et al. Passenger hotspot mutations in cancer driven by APOBEC $3 \mathrm{~A}$ and mesoscale genomic features. Science $\mathbf{3 6 4}$, eaaw2872 (2019) 